\title{
Atypical Choroid Plexus Papilloma
}

National Cancer Institute

\section{Source}

National Cancer Institute. Atypical Choroid Plexus Papilloma. NCI Thesaurus. Code C53686.

A choroid plexus papilloma characterized by increased mitotic activity. 